\title{
Caractéristiques de l'îlot de chaleur urbain à Sfax durant la saison chaude (centre-est de la Tunisie)
}

\author{
Marwen Ghribi ${ }^{1 *}$ et Salem Dahech ${ }^{12}$ \\ ${ }^{1}$ Université de Sfax - Laboratoire SYFACTE, Sfax, Tunisie \\ ${ }^{2}$ Université de Paris, UMR PRODIG UMR8586, Paris, France
}

\begin{abstract}
Résumé - Cette étude s'inscrit dans la continuité des travaux portant sur le climat urbain de Sfax avec une précision spatiale et temporelle. Il s'agit d'une recherche fondamentale apportant certains éléments de réponse à des problématiques d'aménagement, d'économie d'énergie électrique et de prévention du risque sanitaire en cas de forte chaleur. Nous étudions les variations spatiotemporelles des températures de l'air dans l'agglomération de Sfax. En phase nocturne, l'écart thermique centre/campagne atteint son maximum (jusqu'à $\left.7^{\circ} \mathrm{C}\right)$ à partir de minuit. De surcroit, pendant la dernière décennie, les espaces inter-radiaux de la périphérie ont été remarquablement densifiés, suite aux opérations de morcellement, ce qui a engendré l'expansion de la zone chaude à l'échelle de l'agglomération et atténué l'écart thermique entre le centre-ville et la périphérie. De même, la périphérie est l'endroit le plus hétérogène (zones denses, terrains nus, petites exploitations agricoles...), ce qui se traduit par des températures contrastées tributaires de la densité du bâti et des activités anthropiques. Par ailleurs, en phase diurne, la brise de mer rend le littoral, y compris le centre, moins chaud (jusqu'à moins $5^{\circ} \mathrm{C}$ en fonction de la distance) que la périphérie et la zone rurale ouest alors que la brise de terre rend la campagne plus fraîche la nuit.
\end{abstract}

Mots-clés : ICU / température de l'air / mesures fixes et mobiles / variations spatiotemporelles / Sfax.

\begin{abstract}
Characteristics of the urban heat island in Sfax during the hot season (centraleastern Tunisia). This study is a continuation of the work on the urban climate of Sfax with a spatial and temporal precision. It is a fundamental and practice-oriented research bringing certain elements of response to problems of planning, saving electrical energy and prevention of health risk in case of hot weather. This paper study the spatio-temporal variations of the air temperatures in the agglomeration of Sfax, during night. The thermal gap between the centre and the countryside reaches its maximum from midnight. Moreover, during the last decade, the inter-radial areas of the periphery have been remarkably densified, following the operations of fragmentation, which caused the expansion of the hot zone on the scale of the agglomeration and attenuated the thermal difference between the city centre and rural area. Indeed, the periphery is the most heterogeneous area (dense areas, bare land, small farms...), which results in contrasting temperatures depending on the density of buildings and anthropogenic activities. On the other hand, the sea breeze makes the coastline, including the centre, cooler during the day (up to minus $5^{\circ} \mathrm{C}$ depending on the distance) than the periphery and the western rural area, while the land breeze makes the countryside cooler at night.
\end{abstract}

Keywords: UHI / air temperature / fixed and mobile measurements / spatio-temporal variations / Sfax.

$\overline{\text { * Auteur de correspondance }}$ : ghribi310590.marwen@gmail.com

\section{Introduction}

La ville enregistre des températures plus élevées que la campagne voisine notamment durant la nuit; elle forme un îlot de chaleur urbain (ICU) (Oke, 1973 ; Djen, 1992). Fujibe (2011) a montré une hausse moyenne de $3^{\circ} \mathrm{C}$ au niveau des températures annuelles moyennes dans les grandes 
villes japonaises durant le $20^{\text {ème }}$ siècle. Cette hausse, plus significative et plus importante au niveau des températures minimales, a atteint $6^{\circ} \mathrm{C}$ à Tokyo. Les conséquences de l'urbanisation sont, dans certains cas dramatiques particulièrement durant les vagues de chaleur. La coexistence de l'ICU et du réchauffement climatique pourrait conduire dans l'avenir à des situations de canicules urbaines de plus en plus fréquentes (Najjar et al., 2005). La surmortalité, affectant principalement une population vulnérable, augmente à cause des canicules dans les quartiers denses car l'advection d'air chaud s'ajoute à la chaleur excédentaire générée par la ville (surface bâtie et activités anthropiques), ce qui renforce l'aléa (Johnson et Wilson, 2009; Lowe, 2016). De même, l'ICU, particulièrement en été, augmente la consommation en énergie électrique dédiée à la climatisation (Tzoulas et al., 2007 ; Li et al.,2019).

A l'échelle d'un fragment urbain, les paramètres météorologiques varient beaucoup dans l'espace et dans le temps. La température et l'humidité de l'air et le vent varient en fonction de l'encaissement des rues, de leur orientation par rapport au vent dominant, de leur exposition au soleil, des matériaux de construction des bâtiments (couleur, nature...), de la part et de l'état de la végétation, et de la nature des activités anthropiques. Une mosaïque de microclimats se met alors en place (Carrega, 2013). Par conséquent, les données journalières des services météorologiques, généralement mesurées dans les aérodromes, ne peuvent en aucun cas être représentatives des quartiers denses de la ville dans lesquels réside la majeure partie de la population. Une meilleure compréhension de la genèse, de l'évolution du phénomène de l'ICU, de sa spatialisation et de la variation spatiale des températures à l'échelle du quartier permettrait de mieux aménager la ville au futur et renforcer la résilience de la population face aux canicules (Jim, 2004).

A Sfax, une ville de 600000 habitants au centre-est tunisien, les dernières décennies furent marquées par une densification, une extension verticale des surfaces bâties et un étalement urbain accéléré. Ces mutations spatiales avaient des incidences sur la répartition des températures de surface et de l'air (Dahech, 2007 et 2009) induisant la formation d'un climat, particulièrement plus chaud, qualifié d'urbain, au niveau de l'agglomération par rapport à ses alentours dégagés et aux zones rurales (Ghribi et al., 2019). Ce microclimat urbain se manifeste souvent à Sfax en été, car les nuits calmes et claires sont très fréquentes sur la rive sud de la Méditerranée, favorisant les phénomènes radiatifs (Hénia, 1998). De surcroît, l'agglomération de Sfax est implantée sur une vaste plaine côtière homogène et très peu accidentée, ce qui signifie que les contrastes thermiques mesurés entre la ville et ses environs sont essentiellement expliqués par la distance à la mer, la nature du substrat, l'urbanisation et les activités anthropiques (Dahech, 2012). Ces contrastes thermiques, avec une intensité maximale de $1^{\prime}$ ICU atteignant $7^{\circ} \mathrm{C}$, ont été montrés par Dahech $(2007,2009,2012)$ et Dahech et Ghribi (2018) en utilisant la technique des mesures semi-itinérantes et des postes fixes. Le champ thermique et son évolution entre 2002 et 2010 a été caractérisé d'une manière sommaire en privilégiant des points de mesures prises au bord des principaux axes routiers (radiales) vers minuit uniquement. Dans ces études antérieures, l'intensité de l'ICU a été caractérisée à partir des températures minimales quotidiennes.

Dans ce travail, nous explorons d'autres espaces dans l'agglomération et la zone rurale et réalisons des mesures à différentes heures de la nuit pour caractériser l'évolution spatiotemporelle de l'ICU à Sfax et déterminer le moment pendant lequel l'écart centre/périphérie atteint son apogée (intensité maximale) à partir de mesures réalisées instantanément. Ainsi, il est possible de connaitre l'heure à partir de laquelle l'ambiance thermique s'améliore à l'échelle de l'agglomération. Cette étude est donc à la fois fondamentale et appliquée. D'une part, elle apporte une contribution théorique sur le climat urbain de cette ville côtière avec une précision spatiale et temporelle par rapport aux études précédentes menées à Sfax (Dahech, 2007, 2009, 2012), Tunis (Charfi, 2012; Charfi et Dahech, 2018) et Bizerte (Dahech et Charfi, 2017), et dans d'autres villes littorales méditerranéennes comme à Athènes (Livada et al., 2002), 
Thessalonique (Giannaros et Meals, 2012), Barcelone (Salvati et al., 2017). D'autre part, multiples sont les applications possibles des résultats de cette étude. D'abord, ce travail rendra faisable l'étude de l'impact de la variation spatiotemporelle des températures sur l'utilisation de la climatisation électrique et ses conséquences économiques. En effet, les mesures itinérantes réalisées à quatre reprises pendant la nuit, en été, permettraient d'identifier le moment et les espaces où l'utilisation des climatiseurs pourrait devenir inutile. Ensuite, la détermination des zones les moins chaudes, de jour comme de nuit, est incontestablement utile pour mieux aménager le territoire en favorisant autant que possible les sources de fraicheur. Enfin, cette étude peut déboucher sur des recommandations permettant de réduire les impacts sanitaires en cas de vague de chaleur.

Pour apporter des éléments de réponses à ces questionnements, nous utilisons une approche géographique basée sur l'identification des inégalités spatiales et des échelles emboitées à partir de mesures personnelles car l'agglomération est dotée d'une seule station météorologique conventionnelle gérée par l'Institut National de la Météorologie (INM), implantée dans l'aérodrome Sfax-Thyna.

\section{Méthodes et données}

Suivant les modes d'occupation du sol, nous distinguons trois couronnes à Sfax : une première très dense s'étendant sur un rayon de $4 \mathrm{~km}$, une deuxième entre 4 et $11 \mathrm{~km}$ du centre avec une occupation mixte mais plus dense au bord des radiales, et une troisième plus dégagée, dominée par des champs d'oliviers et d'amandiers parsemés d'habitations pavillonnaires et quelques quartiers populaires. Au-delà de cette troisième couronne, le milieu devient rural. Outre les transects centreville/campagne, nous nous intéressons aussi à :

- l'espace inter-radial de la deuxième couronne où nous examinons les incidences de la densification urbaine ;
- un quartier populaire en troisième couronne pour étudier les répercussions des mutations en périphérie sur la température de l'air ;

- la zone rurale, en observant trois sites à différentes distances du centre-ville, à l'ouest de l'agglomération, pour cerner l'intensité de l'ICU suivant les types de temps et l'éloignement au centre.

Plusieurs mesures fixes et mobiles ont été effectuées en vue de mieux étudier les caractéristiques de l'ICU dans l'agglomération de Sfax. En effet, en 2014 et 2017, quatre stations de mesures de type Davis sont installées, simultanément au centre-ville et en zone rurale, en respectant quasiment toutes les normes d'installation conventionnelles (abri hygrothermique à $2 \mathrm{~m}$ de la surface et en respectant la distance requise aux obstacles). Les stations Davis sont de type Vantage Pro2 mesurant la température et l'humidité de l'air, le vent, la pression atmosphérique et les précipitations. Seuls les trois premiers paramètres sont utilisés ici ; leurs résolutions et précisions sont respectivement de l'ordre de 0,1 et $0,5^{\circ} \mathrm{C}$ pour les températures, 1 et $3 \%$ pour l'humidité relative et $0,1 \mathrm{~m} / \mathrm{s}$ et $5 \%$ pour la vitesse du vent et de 1 à $7^{\circ}$ pour la direction d'après le constructeur. Le pas de temps choisi est de 15 min permettant de détecter les variations brusques des paramètres précités. $\mathrm{Au}$ centre-ville, nous avons opté pour le toit d'une école (bâtiment d'un seul étage) car il a été impossible de trouver, à $2 \mathrm{~m}$ de la surface, un site exposé au soleil toute la journée. En milieu rural, en 2014, deux stations ont été implantées à 20 et $13 \mathrm{~km}$ à vol d'oiseau, au sud-ouest du centre et à environ $40 \mathrm{~km}$ à l'ouest en 2017. Le but est de multiplier les stations en milieu rural pour étudier les variations spatiotemporelles de l'intensité de l'ICU en intégrant le paramètre type de temps (figure 1). Pour la zone périurbaine, semi-dégagée, nous avons utilisé les données de l'INM issues de la station fixe de l'aérodrome (figure 1). Le tableau 1 récapitule la localisation, la durée de mesure, et la qualité des données issues des stations météorologiques fixes. Les cinq stations mesurent les températures et l'humidité de l'air, la pression atmosphérique et le vent (direction et vitesse). 
Elles sont étalonnées pour pouvoir comparer leurs données mesurées.

De plus, treize campagnes de mesures itinérantes ont été exécutées pour étudier les contrastes thermiques émergents dans l'agglomération, par un capteur thermohygrométrique de type Testo 665 dont la précision et la résolution sont respectivement de $0,2^{\circ} \mathrm{C}$ et $0,1^{\circ} \mathrm{C}$. L'avantage majeur de ce capteur, outre sa fiabilité, est la réponse rapide de l'ordre de quelques secondes. Le principal transect de mesure est la route de Lafrane ; pour chaque campagne, les mesures itinérantes ont été répétées 4 fois $(22,0,2$ et $4 \mathrm{~h}$ locale) pendant les nuits du 6 juin, 24 juin et 8 juillet 2020 par temps radiatif (transect A sur la figure 1).

Outre le trajet centre-périphérie, une autre campagne $\mathrm{B}$ a été accomplie transversalement au niveau des zones inter-radiales, situées à environ 8 $\mathrm{km}$ du centre-ville, entre les routes de Menzel Chaker et Gremda en passant par El Ain et Lafrane le 1 octobre 2019 et les 6 juin, 24 juin, 8 juillet et 29 juillet 2020 à $1 \mathrm{~h}$ et le 14 juillet 2020 à $3 \mathrm{~h}$ locale. Chaque trajet, opéré en voiture, dure environ $30 \mathrm{~min}$ pour la campagne A et $15 \mathrm{~min}$ pour la campagne $\mathrm{B}$; les faibles variations temporelles de la température pendant ce laps de temps ont été prises en considération.

La campagne $C$, répétée deux fois pendant deux nuits caractérisées par des conditions météorologiques différentes, a été effectuée en courant, pour ventiler le capteur, pendant 15 minutes pour parcourir $2 \mathrm{~km}$, dans un quartier périphérique le 26 août 2019 (vitesse du vent $>3$ $\mathrm{m} / \mathrm{s}$ ) et le 05 septembre 2019 (vitesse du vent $\leq 3$ $\mathrm{m} / \mathrm{s}$ ) à minuit (localisation sur la figure 1). La dernière campagne $\mathrm{D}$ est effectuée le long d'un transect sinueux, d'environ $7 \mathrm{~km}$, allant du centreville au quartier péricentral « El Bahri ». Elle avait pour objectif l'étude de l'impact du volume des bâtiments et de la végétation sur la température.

La variation spatiotemporelle de l'ICU est étudiée suivant les types de temps durant la saison chaude. Trois types de temps se distinguent, en été, à Sfax (station de l'aéroport) d'après Dahech (2007) :
- Un beau temps clair avec alternance brise terremer favorisé par un marais barométrique en surface et un haut géopotentiel en altitude ; c'est le type de temps dominant $(94 \%$ des jours entre le $1^{\text {er }}$ juin et le 14 septembre 2017).

- Un temps clair mais très chaud avec advection, par moment, du sirocco (vent chaud et sec venant du Sahara) aspiré par une dépression sur la Méditerranée ou l'Europe de l'Ouest et remontant une dorsale barométrique en altitude, le vent est faible à modéré et l'air est sec (4 jours soit 3,7\% pendant la période précitée). Les températures maximales et minimales dépassent le percentile 90 $\%$ (période 1981-2010). Ce dernier atteint, pour les températures minimales, $21,7,23,6,24,7$ et $23,6^{\circ} \mathrm{C}$ respectivement aux mois de juin, juillet, aout et septembre, et $33,2,36,36,2$ et $33^{\circ} \mathrm{C}$ pour les maximales. Ce seuil a été utilisé par Ben Boubaker (2010) pour définir les jours avec une très forte chaleur en Tunisie.

- Un temps venté avec un ciel partiellement ou totalement couvert (occurrence de $4 \%$ en été d'après Dahech, 2007) suite à l'infiltration d'une dépression peu creusée sur la Méditerranée, le vent soufflera du nord au nord-est à une vitesse moyenne de $7 \mathrm{~m} / \mathrm{s}$ de jour comme de nuit mais avec des rafales qui dépassent $15 \mathrm{~m} / \mathrm{s}$ (11-13 aout 2017). Rarement, en été, un vent fort soufflera de l'ouest, dans ce cas, l'air sera moins humide (1012 septembre 2017 comme exemple).

\section{Variations spatiotemporelles de I'ICU à Sfax en été d'après les stations fixes}

\subsection{Variations spatiotemporelles en fonction de la distance au centre-ville}

Les données issues des stations fixes sont de très bonne qualité. Les lacunes varient de $0,5 \%$ pour la station rurale 2 , à $7 \%$ au centre-ville (tableau 1). Nous avons examiné et vérifié la cohérence temporelle des données avant leur traitement. Il est judicieux de rappeler que les altitudes des différents sites de mesures restent comparables; son rôle devrait être minime dans l'explication de la variabilité spatiotemporelle des températures. 
Figure 1. (A) Localisation de l'agglomération de SfaxTunisie, (B) des sites des mesures fixes de la température de l'air dans la région de Sfax, (C) des mesures mobiles dans un quartier périphérique et $(D)$ dans l'agglomération (fond, Google Earth 2019). (A) Location of the agglomeration of Sfax-Tunisia, (B) sites of fixed measurements of air temperature in the region of Sfax and (C) mobile measurements in a peripheral district and (D) in the agglomeration (background, Google Earth 2019).

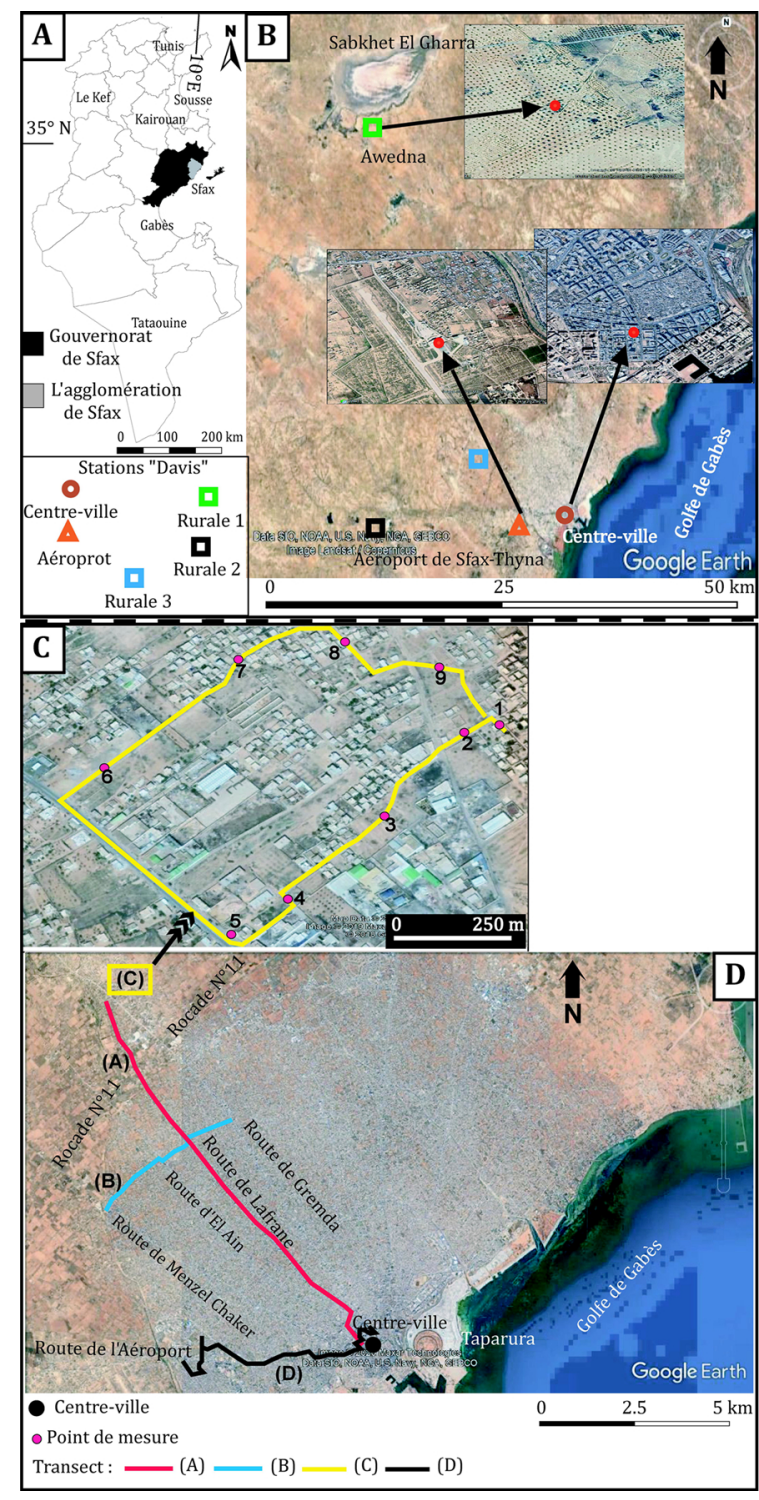

Tableau 1. Réseau des stations météorologiques fixes utilisées. Weather stations network.

\begin{tabular}{|c|c|c|c|c|c|c|c|c|}
\cline { 2 - 9 } \multicolumn{1}{l|}{} & Longitude & Latitude & Altitude & $\begin{array}{c}\text { Distance au } \\
\text { centre-ville }(\mathbf{k m})\end{array}$ & $\begin{array}{c}\text { Distance à la } \\
\text { mer }(\mathbf{k m})\end{array}$ & Période de mesure & Lacunes (\%) & Type \\
\hline Centre-ville & $10^{\circ} 45^{\prime} 22^{\prime \prime}$ & $34^{\circ} 44^{\prime} 55^{\prime \prime}$ & 6 & 0 & 1 & $\begin{array}{c}1 / 6 \text { au } 14 / 9 / 2014 ; \\
1 / 6 \text { au } 14 / 9 / 2017\end{array}$ & 7 & Davis vantage Pro \\
\hline Rurale 1 & $10^{\circ} 33^{\prime} 16^{\prime \prime}$ & $35^{\circ} 05^{\prime} 42^{\prime \prime}$ & 92 & 40 & 34 & $1 / 6$ au $14 / 9 / 2017$ & 6 & Davis vantage Pro \\
\hline Rurale 2 & $10^{\circ} 33^{\prime} 15^{\prime \prime}$ & $34^{\circ} 43^{\prime} 44^{\prime \prime}$ & 75 & 20 & 14 & $1 / 6$ au $14 / 9 / 2014$ & 0,5 & Davis vantage Pro \\
\hline Rurale 3 & $10^{\circ} 37^{\prime} 52^{\prime \prime}$ & $34^{\circ} 47^{\prime} 02^{\prime \prime}$ & 64 & 13 & 14 & $1 / 6$ au $14 / 6 / 2014$ & 5 & Davis vantage Pro \\
\hline Aéroport & $10^{\circ} 41^{\prime} 35^{\prime \prime}$ & $34^{\circ} 43^{\prime} 20^{\prime \prime}$ & 17 & 6 & 6 & $1 / 1 / 1973$ au $1 / 5 / 2020$ & 1 & Réseau national \\
\hline
\end{tabular}


Le cycle quotidien des températures moyennes enregistrées à un pas de temps de $15 \mathrm{mn}$ par quatre stations Davis et à un pas horaire par la station de l'INM durant les mois de juin, juillet et août et la première quinzaine de septembre 2014 et 2017, laisse voir un ICU intense entre minuit et la fin de nuit. En été, les situations radiatives sont omniprésentes et sont favorables à l'apparition des contrastes thermiques liés à la nature du substrat. L'écart entre le centre et la zone rurale 1 atteint un maximum de $4^{\circ} \mathrm{C}$ entre $23 \mathrm{~h}$ et $5 \mathrm{~h}$ du matin (écart négatif, donc la zone rurale est plus fraîche que le centre) (figure 2A). En effet, en milieu urbain, les surfaces artificialisées dominent; elles stockent plus de chaleur pendant la journée à cause de la faiblesse de la part d'énergie solaire utilisée comme chaleur latente, de la géométrie de la ville piégeant les radiations solaires, de la faible ventilation et du volume important des surfaces irradiées. Cependant, les surfaces évaporantes dominent le milieu rural, augmentant la part de la chaleur latente; d'où les quantités restituées de nuit plus faibles que dans la partie centrale dense.

L'écart moyen le plus élevé (pendant la nuit) entre le centre et la zone rurale est influencé par la distance au centre-ville à Sfax. Il atteint $4,5^{\circ} \mathrm{C}$, de $1 \mathrm{~h}$ à $4 \mathrm{~h}$, entre la première station, installée sur le toit d'une école en plein centre-ville (à $2 \mathrm{~m}$ de la surface et à $200 \mathrm{~m}$ de la Médina) et la station rurale 1 (la plus éloignée du centre) située à environ $40 \mathrm{~km}$ de la Médina, en plein champ d'olivier. En s'approchant du centre, aux stations situées à 6,13 et $20 \mathrm{~km}$, l'écart moyen le plus élevé, enregistré entre 2 et $5 \mathrm{~h}$, est respectivement de $2,3,6$ et $3,7^{\circ} \mathrm{C}$ (figure $2 \mathrm{~A}$ ).
Figure 2. (A) Ecart thermique horaire moyen de 4 stations par rapport à la station du centre-ville et (B) écart horaire thermique moyen, maximal et minimal de la station rurale 1 par rapport à la station du centre-ville (moyenne horaire pour la période du $1^{\text {er }}$ juin au 14 septembre des années 2014 et 2017, heure locale, localisation tableau 1 et figure 1). (A) Mean hourly thermal deviation of 4 stations from the downtown station and (B) deviation of rural station 1 from the downtown station (hourly mean for the period from June 1 to September 14 of the years 2014 and 2017, local time, location in table 1 and figure 1). mean, maximum and minimum hourly thermal
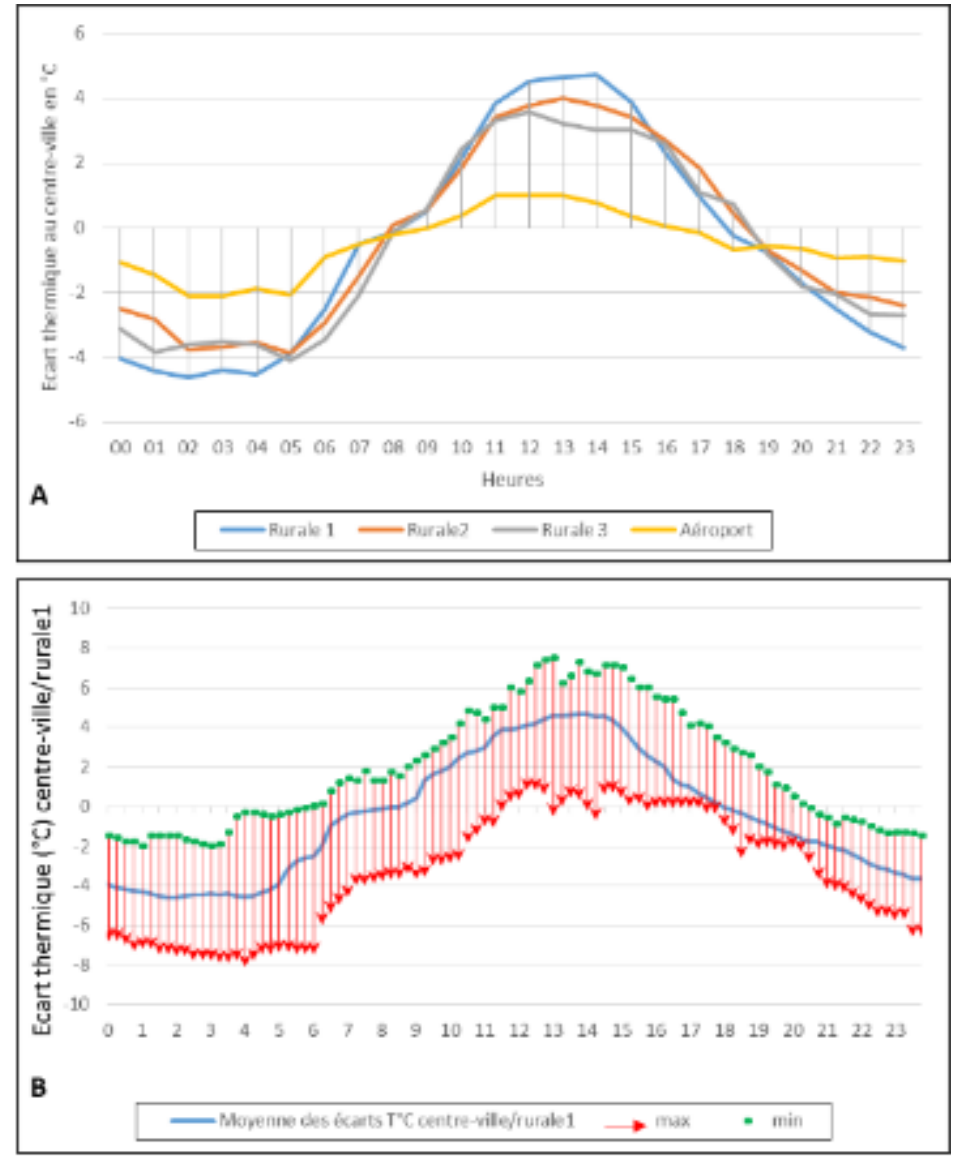

L'écart thermique nocturne le plus faible, par rapport au centre-ville est relevé dans l'aéroport (figures $2 \mathrm{~A}$ et 3 ). L'intensité moyenne de cet écart est d'environ $2^{\circ} \mathrm{C}$ entre 2 et $5 \mathrm{~h}$. Cet écart modéré est dû à la densification des surfaces bâties autour de l'aéroport comme montré dans les travaux de Dahech (2013) et Dahech et Ghribi (2018). De ce fait, la station météorologique de l'aéroport est 
passée d'une station rurale entre 1948 et les années 1980 à une station périurbaine durant les trois dernières décennies.

Contrairement, aux phénomènes radiatifs qui expliquent les contrastes thermiques dans la région de Sfax la nuit, la brise de mer, soufflant du golfe de Gabès, et la position et la distance par rapport au trait de côte influencent la répartition spatiotemporelle des températures pendant la journée (Dahech, 2014). Durant la période estivale retenue, la brise littorale était omniprésente, son occurrence avoisinait $93 \%$. Nous remarquons une alternance entre une brise de mer, le jour, acheminant de l'air frais près de la côte et une brise de terre, la nuit, dont la vitesse ne dépasse pas $2 \mathrm{~m} / \mathrm{s}$ et laissant l'ICU s'installer. En phase diurne, cette brise de mer influence les températures sur le littoral de Sfax (Dahech, 2014). En conséquence, la distance à la mer devient le facteur déterminant pour expliquer la variation des températures au milieu de la journée. Ainsi, les trois stations rurales, plus éloignées de la mer, enregistrent en moyenne, $4^{\circ} \mathrm{C}$ de plus que le centre-ville, à $1 \mathrm{~km}$ de la mer entre $11 \mathrm{~h}$ et $15 \mathrm{~h}$. Dans toutes les stations, l'arrivée de la brise de mer, est accompagnée par une chute des températures et une baisse du contraste thermique par rapport à la station la plus proche de la mer (centre-ville). La chute est observée vers $11 \mathrm{~h}$ à l'aéroport, $12 \mathrm{~h}$ à la station rurale 3 et rurale 2 et à
$15 \mathrm{~h}$ à rurale 1 , situées respectivement à $6,14,14$ et $34 \mathrm{~km}$ de la Méditerranée (figure 4).

\subsection{Variations spatiotemporelles de I'ICU suivant les types de temps}

$\mathrm{Si}$ nous retenons la station du centre-ville comme station de référence urbaine et la station rurale 1 comme référence rurale, nous remarquons que l'intensité maximale et minimale de l'ICU est très variable en fonction des types de temps (figures $2 \mathrm{~B}$ et 3 ).

Durant la période étudiée, l'ICU le plus intense atteint environ $8^{\circ} \mathrm{C}$; il est relevé par temps très chaud et sec (temps détaillé dans la partie méthode). La figure 3 illustre le cycle moyen des températures par temps très chaud durant l'été 2017. Il s'agit de la moyenne des seuls 4 jours recensés pendant la période étudiée. L'intensité maximale de l'ICU est atteinte en fin de nuit, entre 2 et $5 \mathrm{~h}$ du matin à cause du refroidissement notable enregistré dans la zone rurale caractérisée par une faible inertie thermique en fin d'été, très sec. Pendant la journée, la station rurale enregistre une température maximale qui peut dépasser couramment $40^{\circ} \mathrm{C}$ alors que dans le centre et l'aéroport, plus proches de la mer, la brise de mer s'impose souvent car le sirocco possède une faible vitesse : un temps moins torride est observé près de la côte avec des températures aux alentours de $37^{\circ} \mathrm{C}$.

Figure 3. Température horaire de 3 stations par temps très chaud (moyenne horaire de quatre jours durant la période du $1^{\text {er }}$ juin au 14 septembre 2017; données Davis pour les stations Rurale 1 et Centre-ville, et INM pour l'aéroport, heure locale). Hourly temperature for 3 stations under very warm weather (4-day hourly average over the period June 1 to September 14, 2017; Davis data for Rural 1 and Downtown stations and NMI for the airport station, local time).

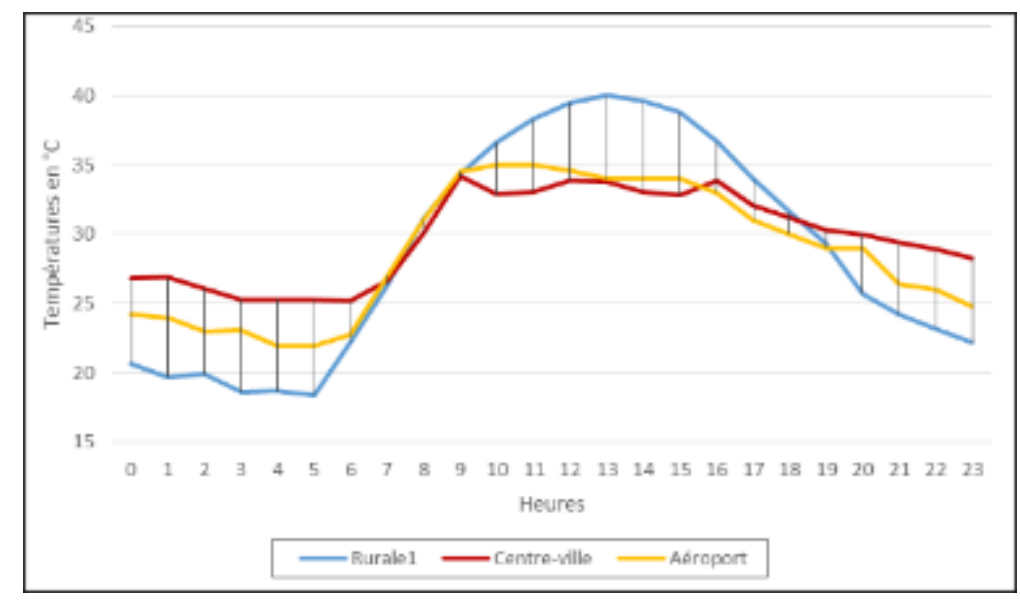

Certes, d'après la littérature (Oke, 1973; Carrega, 1994 ; Cantat, 2004), l'ICU s'estompe par temps couvert et venteux : c'est un phénomène de beau temps. Les mesures fixes réalisées à Sfax confirment cet aspect. Le facteur vent est le plus corrélé avec l'intensité de l'ICU. En effet, à Sfax, 
l'écart thermique au niveau des températures minimales entre la station de l'aérodrome en milieu partiellement dégagé et la station fixe en milieu urbain varie en fonction de la vitesse du vent. La relation entre les deux variables précitées, certes négative, est forte et significative (coefficient de corrélation $r=-0,7$ ).

Quand la vitesse augmente l'écart thermique entre la ville et l'aéroport, la nuit, s'estompe et devient parfois négatif à cause du brassage de l'air. C'est le cas lors d'un épisode venteux du 11 au 13 aout 2017 qui a succédé à une séquence de brise (figure 4B). Nous pouvons retenir un seuil de 4 $\mathrm{m} / \mathrm{s}$ au-dessus duquel la vitesse du vent annule l'ICU d'après les mesures réalisées pendant les étés de 2014 et 2017. Cependant, pendant un épisode de temps radiatif avec déclenchement de brises littorales alternantes, l'écart se creuse entre le centre et l'aéroport. Un ICU intense se met en place, car, le rayonnement solaire direct est élevé le jour et la vitesse de la brise de terre est inférieure à $2 \mathrm{~m} / \mathrm{s}$ la nuit, comme ce fut le cas du 2 au 10 août 2017 (figure 4A), à titre illustratif. Durant cet épisode, la différence au niveau des températures minimales entre le centre-ville et l'aéroport frôle $4^{\circ} \mathrm{C}$ (figure 4B). Pendant la journée, le centre-ville est plus frais avec un écart maximal entre 9 et $11 \mathrm{~h}$, quand la brise de mer se manifeste près de la côte (au centre) et n'atteint pas encore l'aéroport situé à $6 \mathrm{~km}$ de la mer. Cet écart devient faible vers $16 \mathrm{~h}$ quand la vitesse de la brise se renforce.

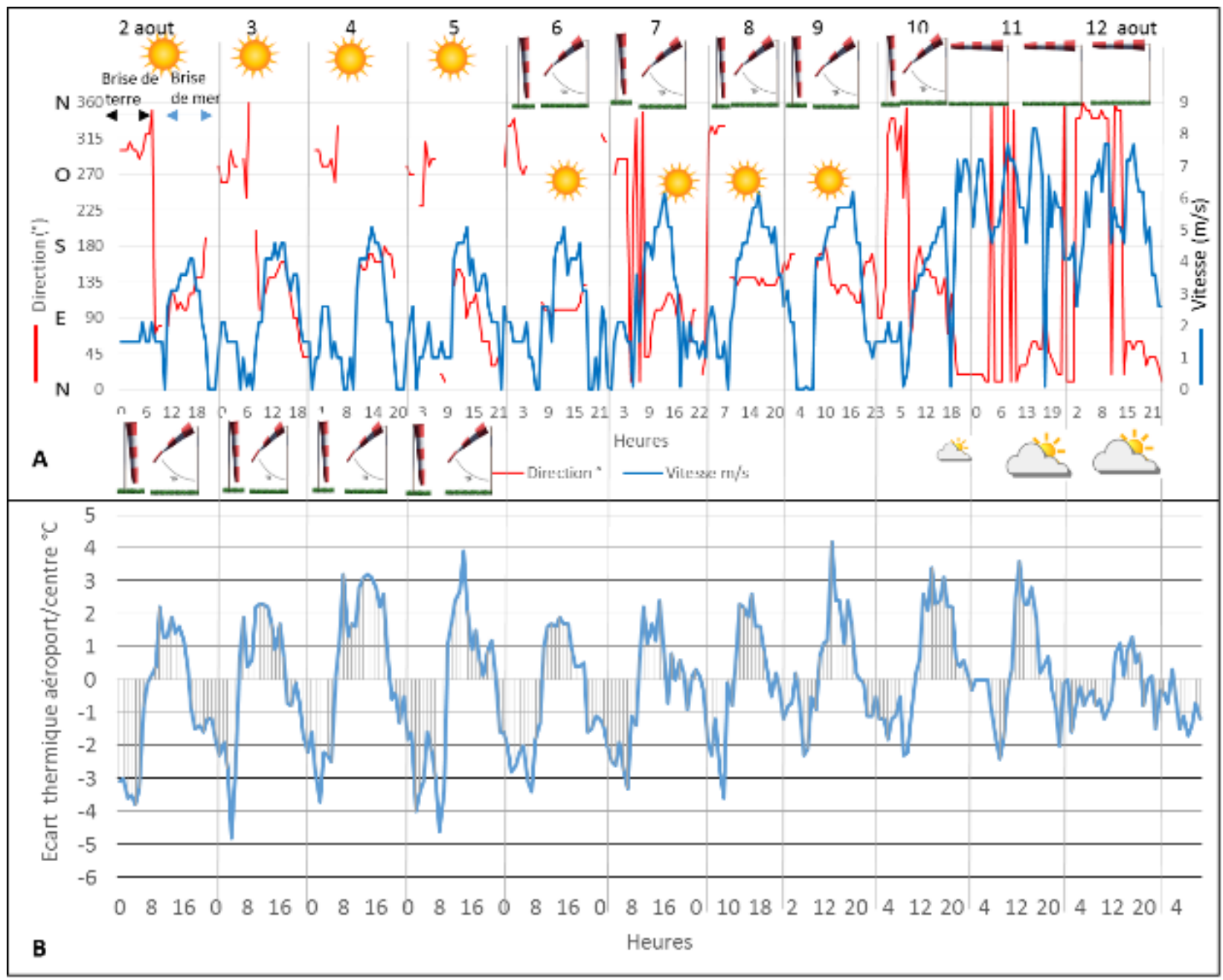

Figure 4. (A) Vent (vitesse et direction) à l'aérodrome de Sfax et (B) écart thermique horaire entre une station fixe en milieu urbain (centre-ville) et la station de l'aéroport du 2 au 13 août 2017 (station Davis et données INM, heure locale). (A) Wind (speed and direction) at Sfax aerodrome and (B) thermal difference between a fixed station in an urban environment (city center) and the airport station from August 2 to 13, 2017 (Davis station and INM data). 
Par rapport à la station rurale 1, durant une journée claire avec alternance entre brise de mer et brise de terre le contraste thermique est plus important que celui par rapport à l'aéroport. La position plus continentale et l'occupation du sol dominée par les oliveraies dans la station rurale explique l'écart thermique qui s'inverse entre le jour et la nuit. L'intensité moyenne de l'ICU pendant ce type de situation est d'environ $5^{\circ} \mathrm{C}$ comme ce fut le cas entre le 27 et le 30 aout 2017 , donné ici à titre d'exemple. Cette intensité est favorisée par le fort ensoleillement le jour et la faible vélocité de la brise de terre en phase nocturne. En effet, la brise de terre ne dépasse pas $1 \mathrm{~m} / \mathrm{s}$ dans les deux stations du centre et rurale 1 où l'anémomètre est installé à environ $7 \mathrm{~m}$ de la surface (figures $5 \mathrm{~A}$ et $\mathrm{B}$ ). Nous remarquons que l'intensité de l'ICU augmente quand l'air est plus chaud et plus sec tel que le cas de la nuit du 28 au 29 aout, durant laquelle l'écart entre le centre et la

Figure 5. Vent (direction et vitesse) aux stations du centre-ville (A), rurale 1 (B) et aéroport (C) du 27 au 30 aout 2017 (données horaires, heure locale, de I'INM et issues des stations Davis, localisation tableau 1 et figure 1). Wind (direction and speed) at the Downtown (A), Rural 1 (B), and Airport (C) stations from August 27 to 30, 2017 (hourly data, local time, from the NMI and Davis monitors, location in table 1 and figure 1). station rurale 1 a atteint $7^{\circ} \mathrm{C}$ vers $4 \mathrm{~h}$ (figure 6). Parallèlement, l'écart thermique le moins élevé est observé pendant la nuit succédant la journée la moins chaude de $1^{\prime}$ épisode étudié, soit $4,5^{\circ} \mathrm{C}$, le 27 août.

Pendant la journée, l'air s'assèche en pénétrant sur le continent avant d'atteindre la station rurale 1 , à $35 \mathrm{~km}$ de la mer, qui demeure très chaude jusqu' à l'arrivée de la brise de mer, attestée par une déviation et un renforcement du vent, vers 15 $\mathrm{h}$ (figure 5B). Les températures chutent rapidement en fin de journée et pendant la nuit à cause de la faible inertie thermique à la campagne sèche et l'effet de la végétation (ombre et part importante de la chaleur latente le jour). Cependant, la proximité de la mer (forte inertie thermique), l'effet urbain et notamment le rayonnement des parois artificielles rendent les nuits plus chaudes en ville et l'amplitude thermique journalière plus faible par rapport au milieu rural.

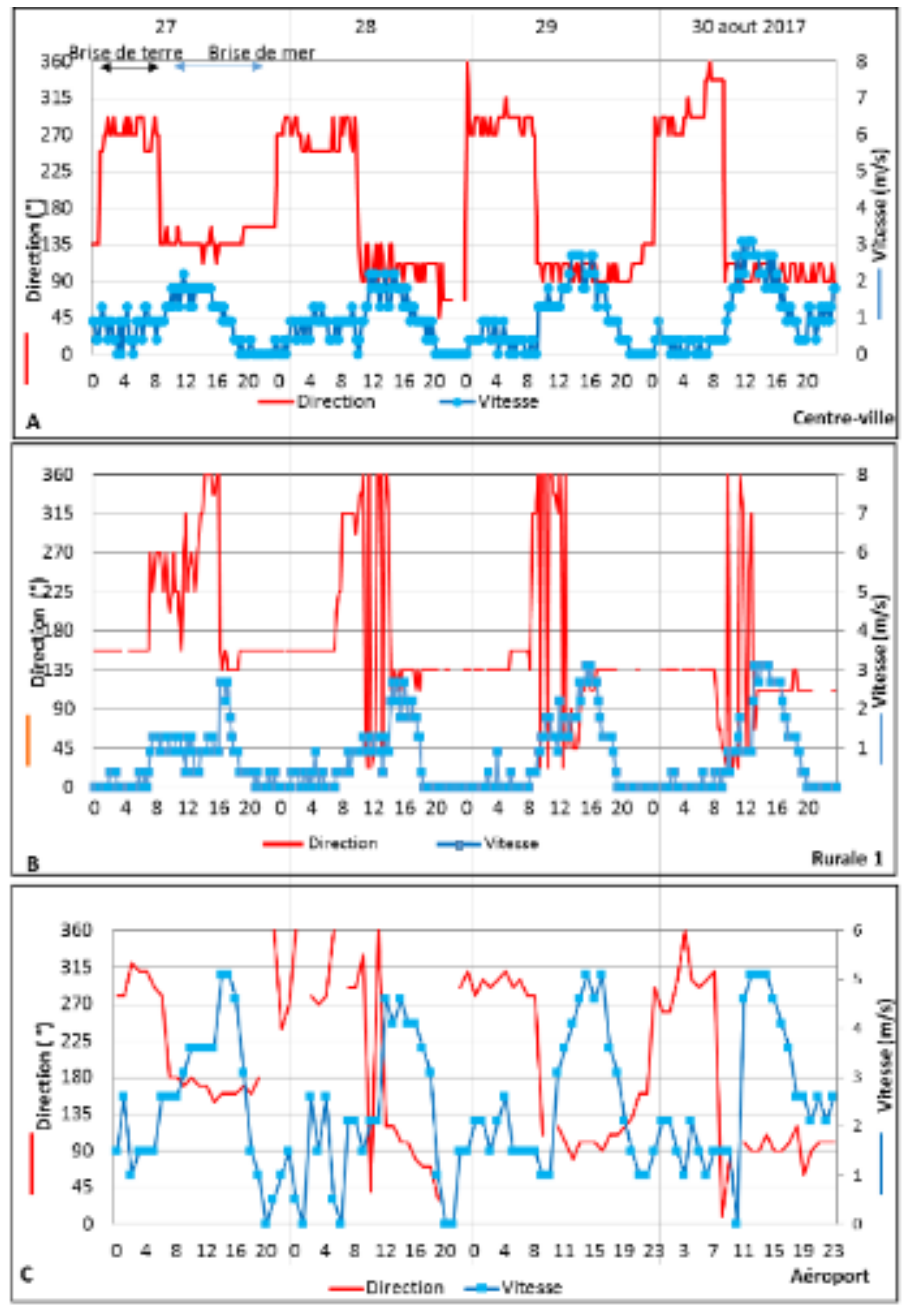


Figure 6. Températures aux stations du centre-ville, rurale 1 et aéroport du 27 au 30 aout 2017 (données horaires, heure locale, données INM et issues des stations Davis, localisation tableau 1 et figure 1). Temperatures at Downtown, Rural 1 and Airport stations from August 27-30, 2017 (hourly data, local time, NMI and Davis stations, location table 1 and figure 1).

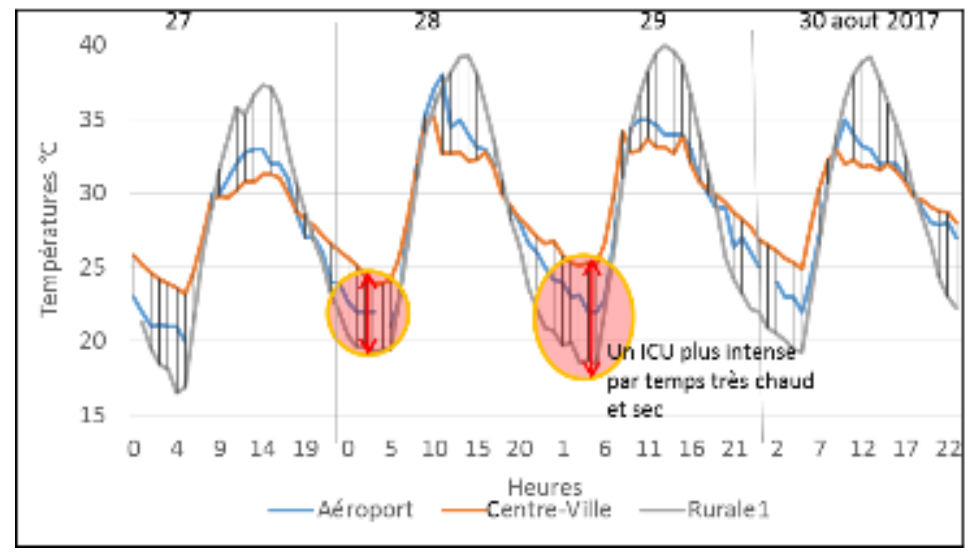

\section{Variations spatiotemporelles des températures de l'air à l'échelle de l'agglomération de Sfax}

\subsection{Champ thermique de l'agglomération de Sfax - Contraste thermique (centre / périphérie)}

Les résultats obtenus par les mesures itinérantes, répétées, des températures de l'air effectuées à $22 \mathrm{~h}$, minuit, $2 \mathrm{~h}$ et $4 \mathrm{~h}$ du matin (heure locale), indiquent un champ thermique remarquablement contrasté. D'une manière générale, les températures de l'air diminuent en s'éloignant du centre-ville vers la périphérie et la zone périurbaine jouxtant la campagne (figures 7 , 8 et 9). La répartition spatiale des températures de l'air est tributaire de la densité du bâti, car les points les plus chauds correspondaient aux zones les plus denses de l'agglomération (Dahech, 2009).

Les mesures effectuées les 6 juin, 24 juin et 8 juillet 2020, montrent un écart thermique de l'ordre de 4 à $6^{\circ} \mathrm{C}$ entre 2 et $4 \mathrm{~h}$ du matin entre le centre et la périphérie le long de la route de Lafrane (figure 7). Les températures diminuent au niveau des terrains nus et des parcelles végétalisées (figures 7 et 8) conformément à la littérature (Charfi et al., 2014). En effet, une baisse de 0,5 à $1{ }^{\circ} \mathrm{C}$ a été enregistrée en passant par les terrains nus, les parcelles végétalisées et les clairières bordant la route (couronne $\mathrm{C}$, figure 8). En fait, ces espaces dégagés, à fort albédo, se refroidissent rapidement la nuit et rafraichissent la mince couche d'air en contact avec la surface, ce qui explique la diminution, parfois, significative des températures au-dessus de ces zones (Avila, 2004). En plus, les parcelles verdoyantes dans la zone périurbaine utilisent une grande partie du rayonnement solaire reçu le jour dans la photosynthèse, la transpiration foliaire et l'évapotranspiration (Luquet, 2002).

Par rapport aux travaux de Dahech (2007 et 2012), les températures sont devenues plus homogènes le long de la route Lafrane jusqu'à 7 $\mathrm{km} \mathrm{du}$ centre à cause de la transformation de plusieurs villas en immeubles de 4 à 8 étages depuis 2010. Nous comptons environ 90 nouveaux immeubles sur ce trajet. L'intensité maximale de l'ICU est enregistrée vers $4 \mathrm{~h}$ du matin quand la brise de terre (fraîche) est plus active en campagne alors qu'elle est quasiment absente au centre-ville à cause de la forte rugosité du substrat. Dans ce dernier, le cumul de la chaleur dégagée par les climatiseurs ${ }^{1}$ et le rayonnent tellurique s'ajoutent à la forte inertie de la mer pour maintenir des températures élevées au centre.

\subsection{Impact du volume des bâtiments et de la végétation sur les températures de l'air dans la partie centrale et péricentrale de l'agglomération de Sfax}

Dans la partie la plus dense, située dans un rayon de $4 \mathrm{~km}$ de la médina, les températures les plus élevées sont mesurées dans le nouveau centre-ville dans le quartier « Nasria ou Sfax Eljadida » dominé par les constructions verticales et volumineuses, ce qui augmentent considérablement les quantités d'énergie stockées et restituées.

${ }^{l}$ D'après le recensement officiel de la population, le taux des ménages équipés par un climatiseur est passé de 5 à $55 \%$ dans 


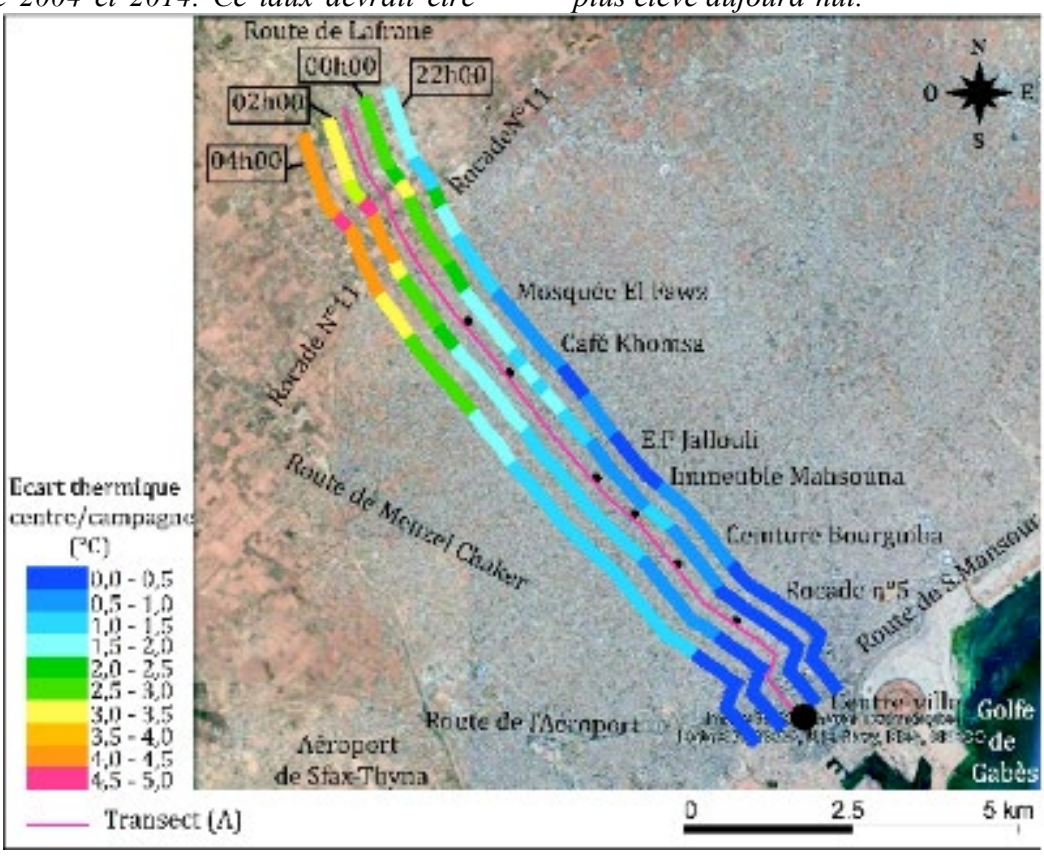

Figure 7. Evolution spatiotemporelle des températures moyennes de l'air dans l'agglomération de Sfax pendant les nuits du 6 juin, 24 juin et 8 juillet 2020 à 22 h, minuit, $2 \mathrm{~h}$ et $4 \mathrm{~h}$ (heure locale) : le cas du transect de Lafrane, (itinéraire A ; figure 1) ; fond : image Google Earth, 2019. Spatiotemporal evolution of the average air temperatures in the agglomeration of Sfax during the nights of June 6, June 24 and July 8, 2020 at 10 p.m., midnight, 2 a.m. and at 4 a.m. in the case of the Lafrane route (campaign A; figure 1); background: Google Earth image, 2019. 
Figure 8. Evolution des températures de l'air en phase nocturne dans l'agglomération de Sfax: d'après les mesures itinérantes du 24 juin 2020 à 2 $\mathrm{h}$ (heure locale), le long du transect de Lafrane: (A) partie centrale, bâti très dense, $(B)$ zone moins dense et $(C)$ zone périphérique dégagée (transect A sur la figure 1 ; fond : image Google Earth 2019). Evolution of air temperatures in the night phase in the agglomeration of Sfax: according to the itinerant measurements of June 24, 2020 at 02:00, along the Lafrane route. (A) Central part very dense, $(B)$ less dense area and $(C)$ peripheral area (itinerary A, figure 1; Background: Google Earth 2019 image).

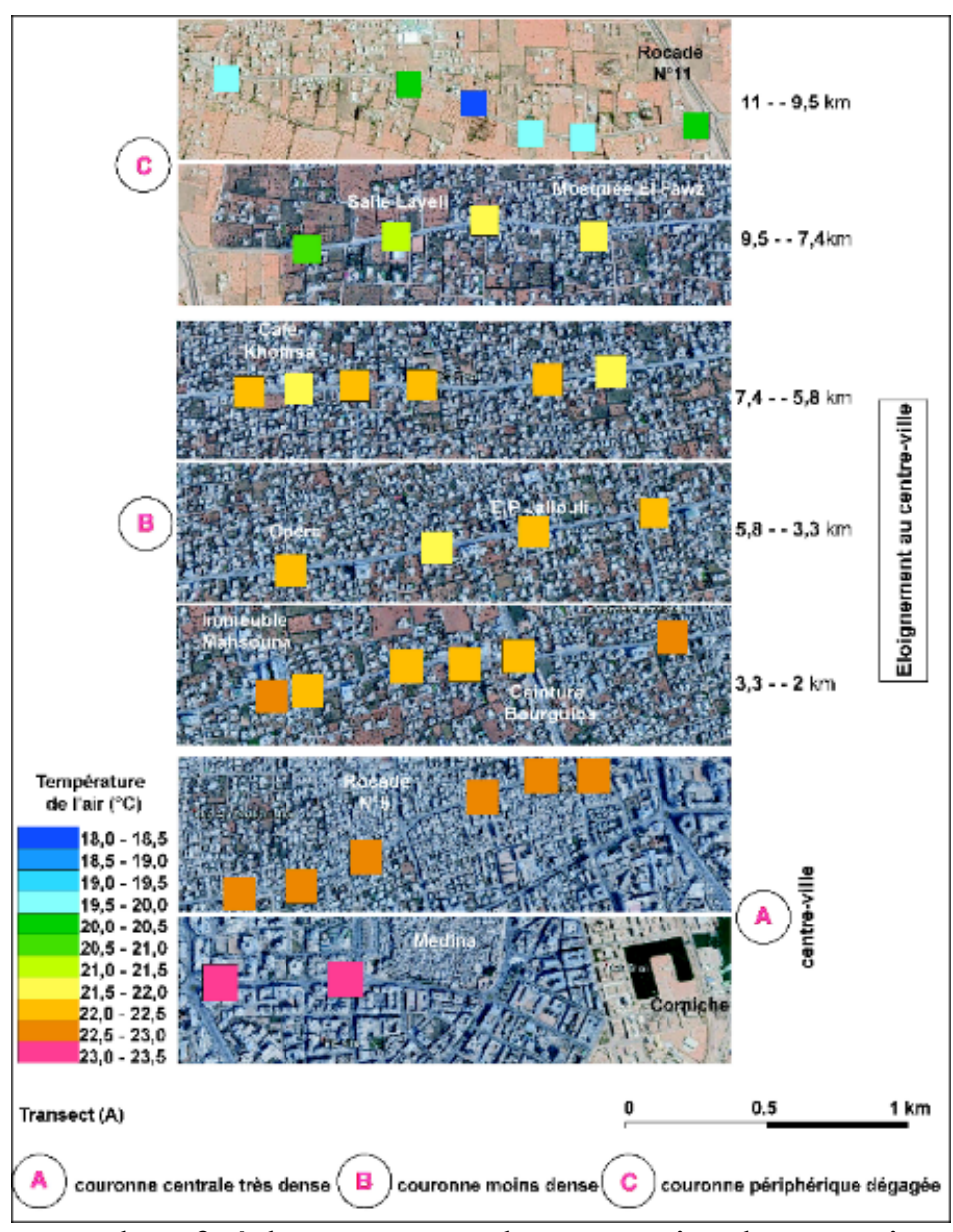

plus fraîche correspond au terrain dégagé situé entre la Faculté des Lettres et des Sciences Humaines (FLSH) et le siège du SAMU (Cité Ennour) (figure 9). Ce terrain nu, près d'Oued El Maou, occupe une petite dépression qui accumule l'air frais.

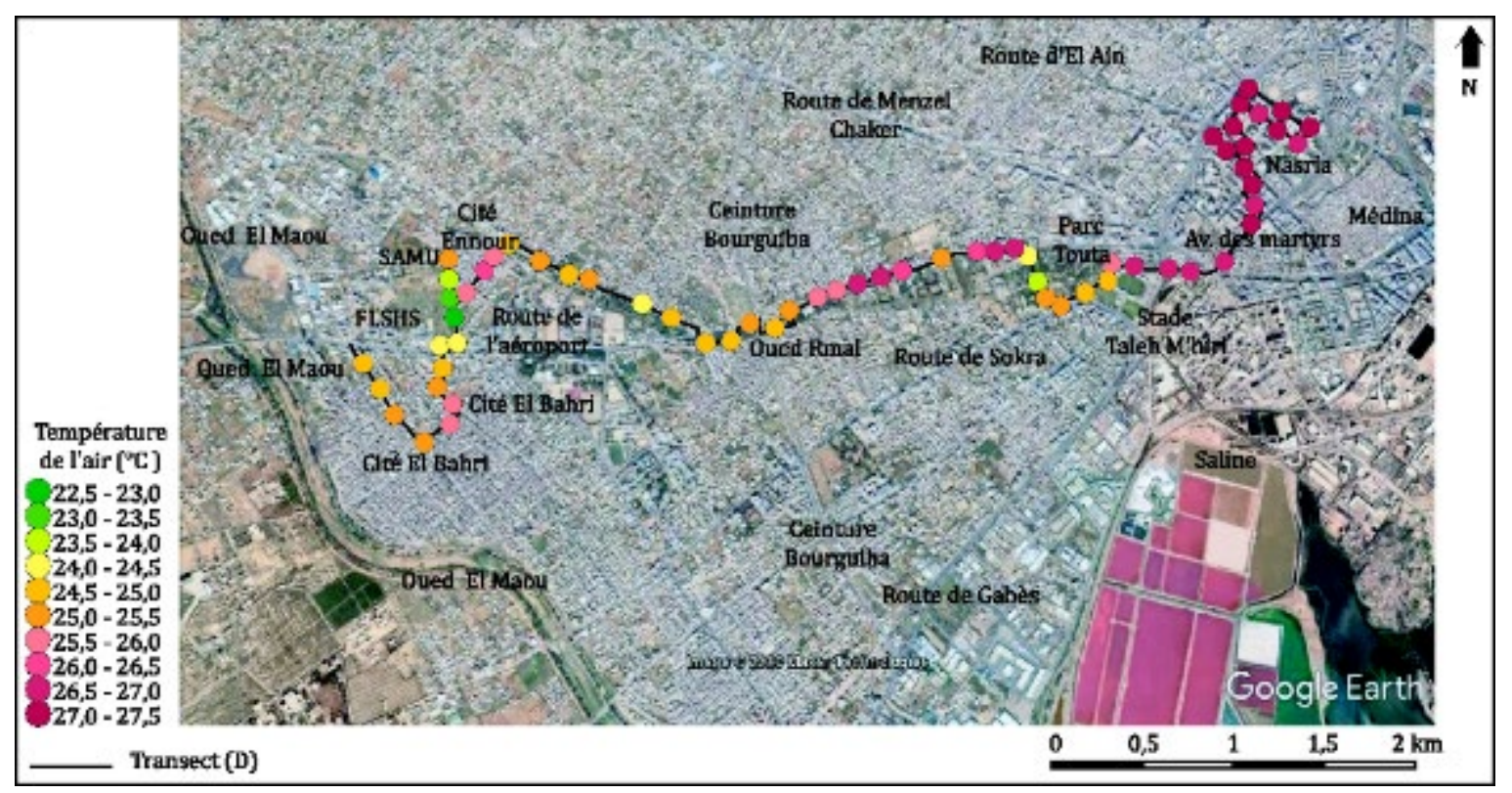


Figure 9. Température de l'air mesurée le long du transect $\mathrm{D}$, zone située entre le centre-ville (Nasria) et Cité El Bahri, le 14 juillet 2020 vers $2 \mathrm{~h}$ (heure locale); fond : image Google Earth 2020. Air temperature measured along the transect D, area between the city center (Nasria) and Cité El Bahri, on July 14, 2020 around 02:00 (local time); background: Google Earth 2020 image.

\subsection{Variations spatiales des températures de l'air dans l'agglomération de Sfax le cas d'une zone inter-radiale et d'un quartier périurbain}

\subsubsection{Rôle de la densification et verticalisation du tissu urbain dans une zone inter-radiale}

La zone inter-radiale est constituée par l'espace situé entre les grandes routes convergeant vers le centre-ville. Elle a été davantage densifiée suite aux mutations récentes que connait cette zone jadis pavillonnaire. Outre la densification du tissu urbain, une verticalisation est observée. Ces mutations avaient des incidences sur la répartition spatiale des températures. Au niveau des endroits inter-radiaux, la répartition spatiale des températures est fortement régie par la nature de l'occupation et de l'utilisation du sol comme le montre les six campagnes de mesures réalisées le long d'un transect de $5 \mathrm{~km}$, au niveau de la délégation de Sfax Sud, par temps radiatif, en été. La température a fluctué presque une vingtaine de fois au cours du trajet, ce qui reflète certainement le changement incessant de la nature du substrat dans cette zone. En fait, tout au long de ce transect, l'espace le moins dense est celui situé entre les routes de Menzel Chaker et El Ain. Puis, à partir $\mathrm{du} 5^{\text {ème }}$ point (B5), en se dirigeant transversalement vers les radiales d'El Ain et Lafrane pour arriver à la route de Gremda au niveau du $20^{\text {ème }}$ point (B20), la densité du bâti augmente sensiblement notamment au niveau de la zone implantée entre les radiales de Lafrane et Gremda (figure 10). Les températures moyennes calculées à partir des six campagnes de mesures itinérantes, réalisées pendant les nuits claires et calmes du $1^{\text {er }}$ octobre 2019, 6 juin, 24 juin, 8 juillet et 29 juillet 2020 vers $1 \mathrm{~h}$ et le 14 juillet 2020 à 3 $\mathrm{h}$, indiquent une forte sensibilité des températures de l'air aux changements de sites (Charfi et Dahech, 2018).

Des micro-îlots de chaleur sont apparus au niveau des zones bâties et artificialisées qui se traduisent par des écarts thermiques, parfois, significatifs par rapport aux terrains dégagés ou végétalisés. Les premiers quatre points (B1 à $\mathrm{B} 4)$, situés au niveau d'une zone faiblement bâtie bordant l'Oued Chaâbouni qui radoucit les températures en canalisant de l'air frais, enregistrent les valeurs les moins élevées. En revanche, les 3 derniers points (B18 à B20) du côté de la radiale de Gremda, zone très dense caractérisée par la présence de constructions verticales et des bâtiments volumineux, enregistrent la température la plus élevée pendant les six campagnes. En fait, l'écart maximal calculé entre les points $\mathrm{B} 2$ et $\mathrm{B} 20$ atteint $3^{\circ} \mathrm{C}$ d'après les mesures effectuées vers $3 \mathrm{~h}$, soit 7 à 8 heures après le coucher du soleil (figure 10B), ce qui corrobore les constatations des mesures fixes «Davis» et itinérantes (ville/campagne) qui indiquent que pendant l'été, le plus fort contraste thermique est observé entre 2 et $4 \mathrm{~h}$ du matin. Au niveau de la zone dense, située entre les points 5 et 20 , une baisse de température entre 0,3 et $0,6^{\circ} \mathrm{C}$ est remarquée en passant par les clairières et les parcelles végétalisées, tels que les points B7, B10 et B16 qui correspondent aux terrains nus ou végétalisés (figures $10 \mathrm{~A}$ et $\mathrm{B}$ ). De plus, les résultats recueillis montrent qu'en plus des surfaces bâties, les points les plus chauds correspondent aux larges chaussées, car le passage au-dessus de ces artères asphaltées et goudronnées (route d'El Ain et Lafrane) s'accompagne, instantanément, par une sensible augmentation des températures (points B9, B14 et B20 ; figures 10A et B). 


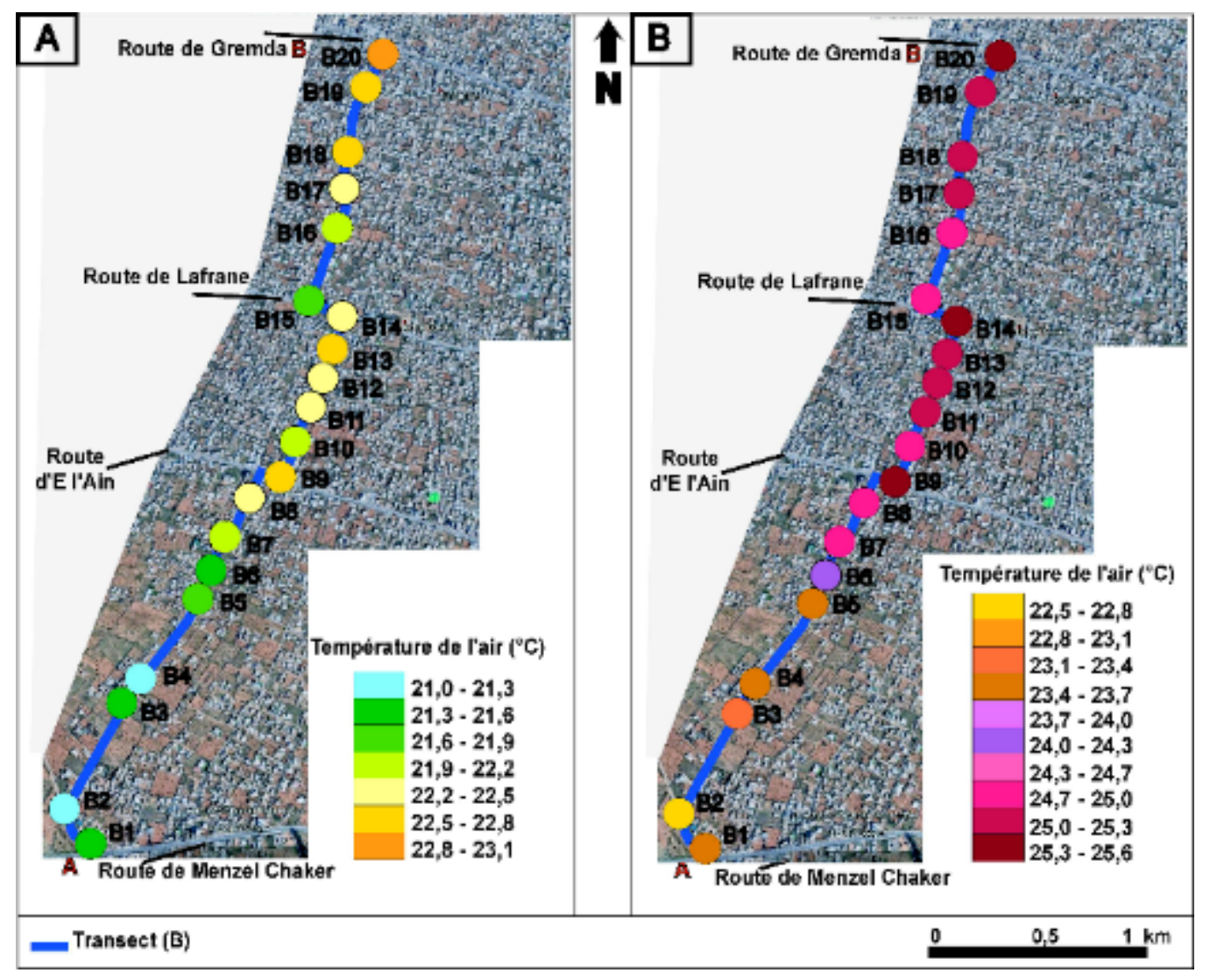

Figure 10. (A) Evolution des températures moyennes de l'air dans une zone inter-radiale de l'agglomération de Sfax, moyenne calculée pour les nuits du $1^{\mathrm{er}}$ octobre 2019, 6 juin, 24 juin, 8 juillet et 29 juillet 2020 à $1 \mathrm{~h}$. (B) Température de l'air mesurée le 14 juillet 2020 à 3 h (heure locale, transect B repéré sur la figure 1; fond : image Google Earth, 2019). Evolution of average air temperatures in an inter-radial zone of the agglomeration of Sfax, average calculated for the nights of October 1, 2019, June 06, June 24, July 08 and July 29, 2020 at 1:00 a.m. (B) Air temperature measured on July 14, 2020 at 3:00 a.m. Background: Google Earth image, 2019.

3.3.2. Rôle des types de temps et de l'urbanisation à l'échelle d'un quartier

A l'échelle d'un quartier périurbain, situé à 11 $\mathrm{km}$ du centre-ville, deux campagnes de mesures itinérantes ont été effectuées en parcourant environ 2 kilomètres, vers minuit, pendant deux types de temps différents. Dans cette zone périurbaine caractérisée par un tissu urbain discontinu, le but était de mettre en exergue l'impact des conditions météorologiques, essentiellement le vent, sur les températures (campagne $\mathrm{C}$ sur la figure 1). En effet, lors de la première campagne réalisée le 26 août 2019 , un écart thermique de $0,6^{\circ} \mathrm{C}$ a été observé entre un îlot bâti (point 8) et une surface dégagée (point 3) pendant cette nuit caractérisée par un vent modéré (> $3 \mathrm{~m} / \mathrm{s}$ ) (figure 9A). La même campagne a été répétée pendant la nuit claire et calme du 5 septembre 2019 (vitesse du vent $\leq 2 \mathrm{~m} / \mathrm{s}$ ) (figure 11B), lors de laquelle nous avons détecté plus de contrastes. Dans ce fragment urbain, l'écart a atteint $1^{\circ} \mathrm{C}$ entre le point 1 et le point 10 (figure 11B). En effet, cette variation thermique à micro-échelle est principalement due à la densité du bâti : les secteurs les plus densément construits paraissaient plus chauds que les espaces vides et dégagés. 
Figure 11. Variations spatiales des températures de l'air dans un quartier périurbain (A) la nuit du 26 aout 2019, (B) la nuit du 5 septembre 2019 vers minuit. Fond: Image Google Earth, 2019. Spatial variations in air temperatures in a peri-urban district $(A)$ on the night of August 26, 2019, (B) on the night of September 05, 2019 around midnight. Google Earth image, 2019.

\section{Conclusion}

Cette étude montre à la fois l'impact des brises littorales, de l'occupation du sol et certaines activités humaines sur la variation spatiotemporelle des températures de l'air durant la saison chaude, à différentes échelles emboitées. Le recours aux mesures itinérantes, répétées plusieurs fois durant la même nuit et la mise en place de stations fixes dans trois sites ruraux, apportent des résultats inédits dans l'espace géographique étudié concernant la variation spatiotemporelle de l'intensité maximale de l'ICU.

L'artificialisation incessante de l'espace urbain de Sfax a donné naissance à un microclimat distingué par l'émergence d'un ICU pendant les nuits radiatives. L'intensité de l'ICU dépend d'abord de la référence rurale. En s'éloignant du centre et de la mer les températures baissent considérablement la nuit en zone rurale. Une intensité maximale de $1^{\prime} \mathrm{ICU}$ d'environ $7^{\circ} \mathrm{C}$ est

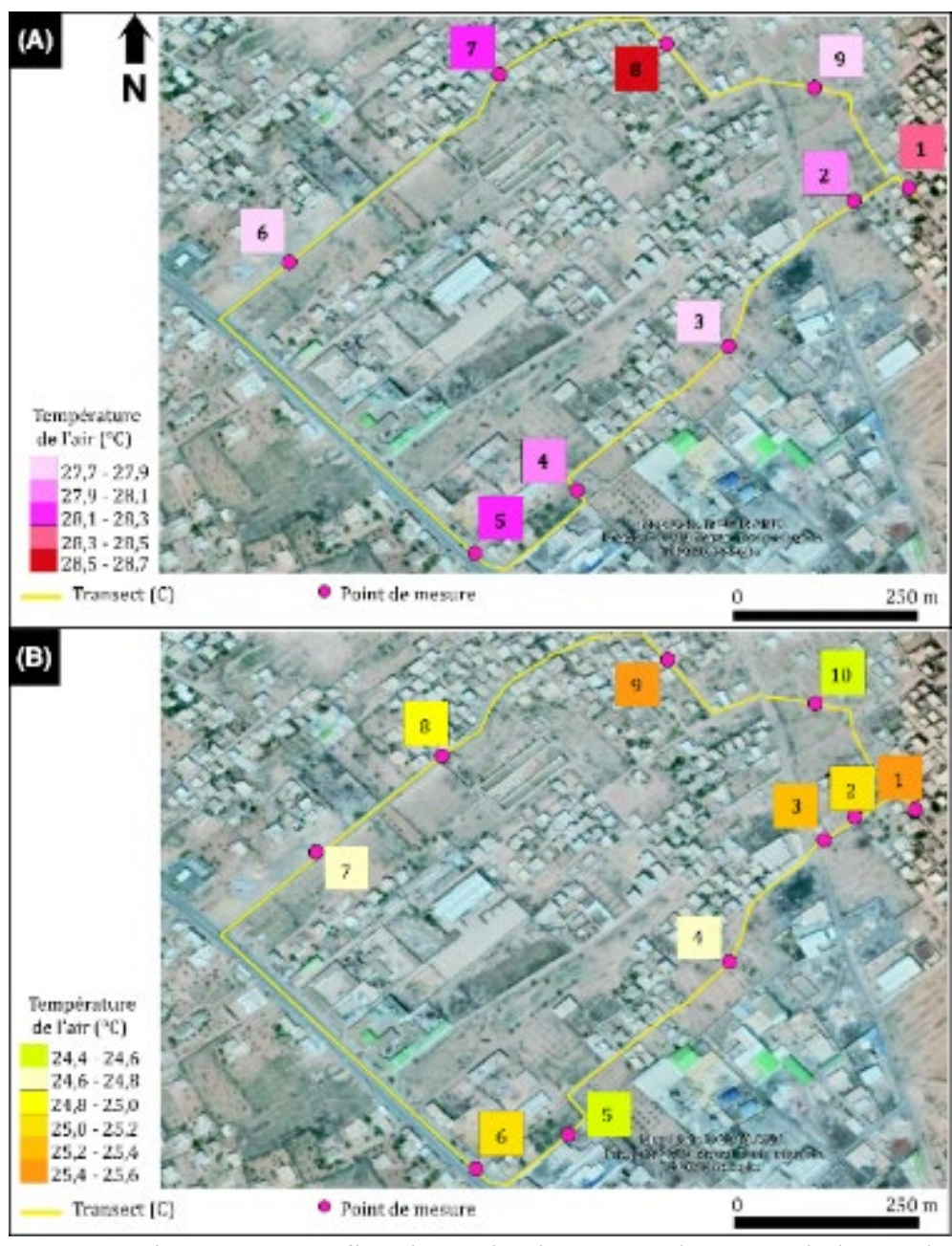

observée en fin de nuit de 2 à $4 \mathrm{~h}$ entre juin et la première quinzaine de septembre, par temps très chaud. Elle est plus précoce au mois d'octobre et plus modérée par temps radiatif caractérisé par l'alternance entre brise de mer et brise de terre. Outre l'écart centre-ville/campagne, des contrastes thermiques, plus modérés, sont également observés au niveau de la périphérie entre les îlots bâtis et les espaces dégagés. La densification qu'a connue la zone périurbaine pendant les dernières années s'est accompagnée par l'émergence de certains îlots chauds de part et d'autre des routes principales, comme le cas le long de la route Lafrane. La brise de terre, plus active en zone dégagée semble rafraîchir la campagne, alors que dans la partie dense, la chaleur anthropogénique, notamment celle rejetée par les climatiseurs, renforce l'ICU.

Le contraste ville/campagne devient très faible quand la vitesse du vent dépasse $4 \mathrm{~m} / \mathrm{s}$ et peut totalement disparaître pendant les nuits perturbées (vent fort, $>8 \mathrm{~m} / \mathrm{s}$ ). De plus, la nébulosité, même 
pendant les nuits calmes, peut ralentir le rafraichissement dans les zones périphériques, en maintenant les rayonnements telluriques dans la basse couche de l'atmosphère, et diminuer évidemment l'écart centre/campagne.

Une baisse d'environ $3^{\circ} \mathrm{C}$ dans le jardin public Touta indique 1'importance des parcs urbains arrosés pour réduire l'ICU. Par ailleurs, l'expansion accrue de la surface chaude dans la ville de Sfax engendrerait certainement l'extension des îlots d'inconfort thermique à l'échelle de l'agglomération et augmenterait évidemment le recours à la climatisation électrique et la consommation énergétique pendant la période chaude de l'année (Ghribi et al., 2019). L'usage de la climatisation électrique est devenu fréquent dans la partie dense de l'agglomération; il explique, en partie, la quasi-homogénéité du champ thermique dans un rayon de $7 \mathrm{~km}$ du centre-ville. Dans la zone périphérique et les quartiers moins denses, la nuit, la population pourrait se passer de la climatisation électrique car la brise de terre et la faible densité du tissu urbain favorisent la baisse relative des températures. Dans les quartiers populaires denses, situés près du centre-ville, le faible niveau socio-économique est un paramètre accentuant la vulnérabilité de la population face à la forte chaleur estivale. Les répercussions sanitaires de cet aléa devraient être non négligeables pour la population sensible. L'exposition à des températures élevées et le facteur socio-économique sont des éléments à prendre en considération dans l'élaboration du plan de prévention des risques liés aux canicules à Sfax.

Les travaux en cours de finalisation portent sur la variation spatiotemporelle de la consommation de l'énergie électrique, en été, en fonction du degré $\mathrm{du}$ confort thermique dans l'agglomération de Sfax. Des différences entre le champ thermique et la répartition des indices de confort à l'échelle de l'agglomération, suivant les types de temps, pourraient influencer la consommation de l'énergie électrique dédiée à la climatisation.

Remerciements : Nous tenons à remercier très chaleureusement monsieur Sami Charfi, enseignantchercheur à l'université de Sfax / laboratoire SYFACTE, de nous avoir prêté les capteurs thermo- hygrométriques. Les auteurs adressent leurs vifs remerciements à Mohamed Chebli, Hamdi Euchi et Wassim Madani qui ont participé aux mesures itinérantes. Les travaux de terrains ont été financés, en partie, par le projet PHC Maghreb "Îlots de chaleur urbains dans les villes du sud de la Méditerranée : diagnostic et résilience" 39361WA.

\section{Bibliographie}

Avila F., 2004. Les climats thermiques locaux : influence du substrat et de la topographie sur la formation des climats thermiques locaux dans une région de plaine du sud de l'Aquitaine (Moyen-Adour et forêt landaise). Revue géographique des Pyrénées et du Sud-Ouest. 18, 131-142.

Ben Boubaker H., 2010. Les paroxysmes climato-thermiques en Tunisie : approche méthodologique et étude de cas. Climatologie, 7, 57-87.

Cantat O., 2004. L'îlot de chaleur urbain parisien selon les types de temps. Norois, ${ }^{\circ} 191,2,75-105$.

Carrega P., 2013. Le climat urbain de Nice en milieu géographique contrasté : Synthèse par approche inductive. Climatologie, 10, 9-36.

Charfi S., 2012. Le comportement spatio-temporel de la température dans l'agglomération de Tunis. Thèse, Université de Tunis et Université de Nice, 308 p.

Charfi S., Krout H., Dahech S., 2014. Vegetation in the urban area of Tunis: Study of impacts on temperature and light. Climatologie, 11, 72-78.

Charfi S. et Dahech S., 2018. Cartographie des températures à Tunis par modélisation statistique et télédétection. Mappmonde, 123, 2018, 1-16.

Dahech S., 2007. Le vent à Sfax (Tunisie), impacts sur le climat et la pollution atmosphérique. Thèse de doctorat de l'Université Paris VII, 309 p + annexes.

Dahech S., 2009. Aggravation du réchauffement en milieu urbain à Sfax (Tunisie). Fifth Urban Research Symposium 2009, Marseille, 27 juin- ${ }^{\text {er }}$ juillet.

Dahech S., 2012. Evolution de la répartition spatiale des températures de l'air et de surface dans l'agglomération de Sfax (1975-2010) et impact sur la consommation d'énergie durant la saison chaude. Climatologie, numéro spécial 'Climats et changement climatique dans les villes',11-33.

Dahech S., 2013. Le réchauffement contemporain en Tunisie (1960-2012) : rôle de la circulation atmosphérique et de l'urbanisation. Journées de la Commission Climat et Société du Comité National Français de Géographie, 14-16 mars, Epernay, 68-88.

Dahech S., 2014. Impact de la brise de mer sur le confort thermique au Maghreb oriental durant la saison chaude, Cybergeo : European Journal of Geography [En ligne], Environnement, Nature, Paysage, document 695, mis en 
ligne le 02 décembre 2014, consulté le 11 septembre 2017. DOI : 10.4000/cybergeo.26555

Dahech S. et Charfi S., 2017. Aspects topoclimatiques de Bizerte (NE de la Tunisie): Ilot de chaleur et brises thermiques. Les Impromptus, 138-158.

Dahech S. et Ghribi M., 2018. Réchauffement climatique en ville et ses répercussions énergétiques. Cas de l'agglomération de Sfax (centre-est de la Tunisie). Méditerranée, 128, 29-38.

Djen C. S., 1992. The urban climate of Shanghai. Atmospheric Environment, 26, 9-15.

Fujibe F., 2011. Urban warming in Japanese cities and its relation to climate change monitoring. Int. J. Climatol, 31, 162-173.

Fung W., Lam K., Hung W., Pang S. et Lee Y. L., 2006. Impact of Urban Temperature on Energy Consumption of Hong Kong. Energy, 31, 2623-2637.

Ghribi M., Euchi H., Dahech S., 2019. Inconfort thermique et olfactif dans l'agglomération de Sfax : perception dans les quartiers populaires de Sfax-Ouest (Tunisie). Publications de l'AIC, 359-364.

Giannaros T. et Meals D., 2012. Study of the urban heat island in a coastal Mediterranean City: The case study of Thessaloniki, Greece. Atmospheric Research 18, 103-120. https://doi.org/10.1016/j.atmosres.2012.06.006.

Henia L., 1998. Les situations anticycloniques en Tunisie. Publications de l'Association Internationale de Climatologie, 11, 166-174.

Jim C. Y., 2004. Green-space preservation and allocation for sustainable greening of compact cities. Cities, 21, 311-320.

Johnson D. P. et Wilson J. S., 2009. The socio-spatial dynamics of extreme urban heat events: the case of heat- related deaths in Philadelphia. Applied geography, 29, 419434.

Li X., Zhou Y., Yu S., Jia G., Li H., Li W., 2019. Urban heat island impacts on building energy consumption: A review of approaches and findings. Energy, 174, 407-419.

Livada I., Santamouris M., Niachou K., Papanikolaou N., Mihalakakou G., 2002. Determination of places in the great Athens area where the heat island effect is observed. Theor. Appl. Climatol. 71, 219-230. doi:10.1007/s007040200006.

Lowe S. A., 2016. An energy and mortality impact assessment of the urban heat island inthe US. Environmental Impact Assessment Review, 56, 139-144.

Luquet D., 2002. Suivi de l'état hydrique des plantes par infrarouge thermique : analyse expérimentale et modélisation $3 D$ de la variabilité thermique au sein d'une culture en rang de cotonniers. Thèse de doctorat, Institut National Agronomique, Paris, INA-PG, $166 \mathrm{p}$.

Najjar G., Kastendeuch P., Ringenbach N., Colin J. R., Stoll M. P., Nerry F., Bernard J., De Hatten A., Luhahe R. et Viville D., 2005. Bilans radiatifs et énergie dans un cayon urbain. Annales de l'AIC, 2, 41-54.

Oke T. R., 1973. City size and heat urban island. Atmospheric Environment, 7, 769-779.

Salvati A., Coch Roura H., Cecere C., 2017. Assessing the urban heat island and its energy impact on residential buildings in Mediterranean climate: Barcelona case study. Energy and Buildings, 146, 38-54. https://doi.org/10.1016/j.enbuild.2017.04.025.

Tzoulas K. et al., 2007. Promoting ecosystem and human health in urban areas using Green Infrastructure: A literature review. Landscape and Urban Planning, 81, 3, 167-178.

Citation de l'article : Ghribi M. et Dahech S., 2020. Caractéristiques de l'îlot de chaleur urbain à Sfax durant la saison chaude (centre-est de la Tunisie). Climatologie, 17, 7. 